\title{
La recherche du boson de Higgs au Tevatron
}

Gregorio Bernardi (gregorio@in2p3.fr)

LPNHE, IN2P3 et Universités Paris VI et VII, 4 place Jussieu, 75252 Paris Cedex 05

Après une introduction

au boson de Higgs,

nous présentons sa recherche

dans le cadre du Modèle

Standard par les expériences

CDF et DØ auprès

du collisionneur Tevatron

du Fermilab (USA).

Avec les données actuellement

analysées, le Tevatron exclut

que la masse du boson de

Higgs du Modèle Standard

soit entre 160 et $170 \mathrm{GeV} / \mathrm{c}^{2}$

(1). L'analyse des prochaines

données couvrira la zone

entre 115 et $160 \mathrm{GeV} / \mathrm{c}^{2}$,

favorisée par la théorie

et par les indications

expérimentales indirectes.

Nous concluons sur les

perspectives de découverte

de cette particule mystérieuse

dans les deux ou trois

prochaines années.

(1) La masse du proton est d'environ $1 \mathrm{GeV} / \mathrm{c}^{2}$; dans la suite, on posera $c^{2}=1$.
La physique fondamentale, de l'infiniment petit à l'infiniment grand, se trouve aujourd'hui dans une situation paradoxale. D'un côté, il existe une théorie des particules élémentaires, le Modèle Standard (MS), achevée au milieu des années 70 et qui, depuis, n'a jamais été mise en défaut malgré un très grand nombre de tests expérimentaux de plus en plus détaillés. De l'autre, les physiciens sont conscients des limites de cette théorie, tant sur le plan conceptuel que pour appréhender l'univers dans son ensemble, comme le montre de manière éclatante l'incompréhension de la nature de la matière noire et de l'énergie noire [1]. Bien que le Modèle Standard reste inviolé à ce jour, le nombre de questions qu'il soulève permet de l'attaquer sous plusieurs angles. Puisqu'il relie par les interactions faible et électromagnétique les leptons (tel l'électron) et les quarks, la question de l'existence de trois familles de particules élémentaires, ne différant que par les masses des deux quarks et des deux leptons qui les constituent, se trouve tout de suite posée (voir tableau ci-dessous).

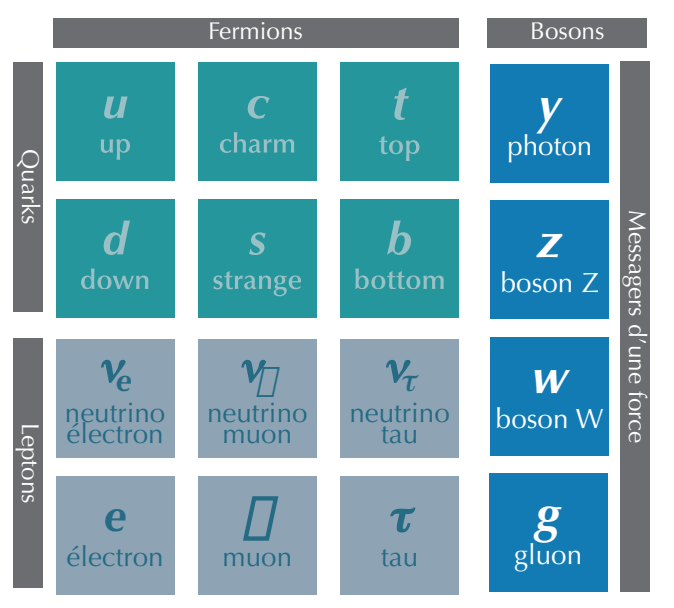

Tableau représentant les particules du Modèle Standard, à l'exception du boson de Higgs qui y joue un rôle particulier, puisqu'il intervient pour briser la symétrie électrofaible et donner une masse à toutes ces particules élémentaires (excepté le photon et les gluons). Chacune de ces particules a une antiparticule associée (notée par un surlignage, par exemple $\bar{p}$ pour l'antiproton). Rappelons que le proton et le neutron sont des combinaisons triplettes, " uud » et " udd » respectivement, soumises à l'interaction forte, par l'intermédiaire des gluons.
Seule la première famille, constituée des particules les plus légères (colonne de gauche du tableau), est présente dans les atomes formant les molécules de notre monde ; alors pourquoi d'autres familles, qui n'apparaissent que fugitivement à haute énergie ?

\section{Le problème de la masse des particules élémentaires et le mécanisme de Higgs}

Les valeurs relatives des masses des particules entre familles, ou à l'intérieur d'une même famille, suivent aussi un schéma que nous sommes incapables d'expliquer. Pour essayer de comprendre cette caractéristique fondamentale des particules, leur masse, il faut revenir à la théorie quantique sous-jacente au Modèle Standard, en particulier celle qui permet de décrire l'unification des forces faible et électromagnétique, en une force électrofaible. Cette théorie n'autorise pas dans son état fondamental l'existence de masses non nulles pour les particules élémentaires. Cela posait bien sûr problème pour la description des particules existantes, lorsque la théorie fut avancée à la fin des années 60 .

L'avancée conceptuelle qui permit à cette théorie de s'imposer fut la compréhension que s'il existait une particule $H$ supplémentaire possédant des caractéristiques singulières, celle-ci entraînerait dans les premiers instants après le Big Bang la brisure de la symétrie qui garde unifiées les forces faible et électromagnétique à haute énergie. Cette brisure rendrait massives toutes les particules élémentaires, la particule $\mathrm{H}$ se transformant en une particule observable et également de masse non nulle :le boson de Higgs. Cette brisure "spontanée " de la symétrie est le "mécanisme de Higgs ", du nom de Peter Higgs, le physicien qui fut un des premiers à le formuler (avec François Englert et Robert Brout). Dans le mécanisme de Higgs, les bosons de jauge $W$ et $\mathrm{Z}$ de l'interaction électrofaible acquièrent des masses respectives d'environ 80 et $91 \mathrm{GeV}$, directement à partir du champ quantique initial, tandis que le photon reste de masse nulle. Les quarks et les leptons acquièrent leur masse d'une autre manière, par interaction avec le champ omniprésent associé au boson de Higgs. Bien que ce mécanisme puisse sembler 
" incroyable ", les brisures spontanées de symétrie existent souvent dans la nature : c'est, par exemple, le cas du ferromagnétisme, qui engendre un alignement de tous les moments magnétiques élémentaires des atomes dans une direction donnée, alors que le système avait une symétrie isotrope avant la brisure.

\section{La recherche du boson de Higgs}

Le mécanisme ne peut cependant pas prédire la valeur des masses des particules, et la recherche du boson de Higgs se poursuit depuis les années 70, sans succès jusqu'à présent. Comme il n'a pas été observé dans le collisionneur LEP du CERN (arrêté en 2001), ceci montre que sa masse $m_{H}$ est supérieure à $114 \mathrm{GeV}$ [2]. Par ailleurs, des relations théoriques indirectes dans le Modèle Standard, relient la masse du boson de Higgs à celles du boson W et du quark top, ainsi qu'à d'autres observables expérimentales mesurées, qui permettent d'être sûr que $\mathrm{m}_{\mathrm{H}}$ est inférieure à environ $185 \mathrm{GeV}$. En effet, la probabilité pour que ce soit le cas est supérieure à 95\% (l'on définit alors ce résultat comme une limite supérieure à 95\% de niveau de confiance, C.L.). En résumé, ces contraintes montrent que $\mathrm{m}_{\mathrm{H}}$ doit se situer entre 115 et $185 \mathrm{GeV}$.

Le boson de Higgs pourrait donc être vu dans les deux ou trois années qui viennent par les collaborations CDF et D $\varnothing \mathrm{du}$ Tevatron, le collisionneur proton-antiproton du Laboratoire de Fermi, près de Chicago (voir encadré). Les performances du Tevatron ont progressé de manière continue depuis 2003. Les améliorations déjà réalisées permettent de prévoir une quantité suffisante de données (soit $\mathrm{L}=8 \mathrm{fb}^{-1}$, si on utilise l'unité qui permet de compter le nombre de collisions accumulées pendant la durée de la prise de données) à la fin des expériences, prévue en 2010, pour envisager une découverte du boson de Higgs, si les analyses atteignent elles aussi le degré de performances requis.

\section{Le Tevatron au Fermilab}

Le Laboratoire de Fermi (Fermi National Accelerator Laboratory) est un laboratoire national américain, situé à $70 \mathrm{~km}$ à l'ouest de Chicago (Illinois), dédié à la recherche fondamentale en physique des hautes énergies et disciplines associées. Inauguré en 1967, il dépend du Département de l'Énergie (DOE) et est ouvert aux chercheurs de toutes nationalités.

Le Fermilab a effectué des découvertes majeures en physique des particules : le quark bottom (1977) et le quark top (1995), composantes du Modèle Standard, puis le neutrino tau (2000).

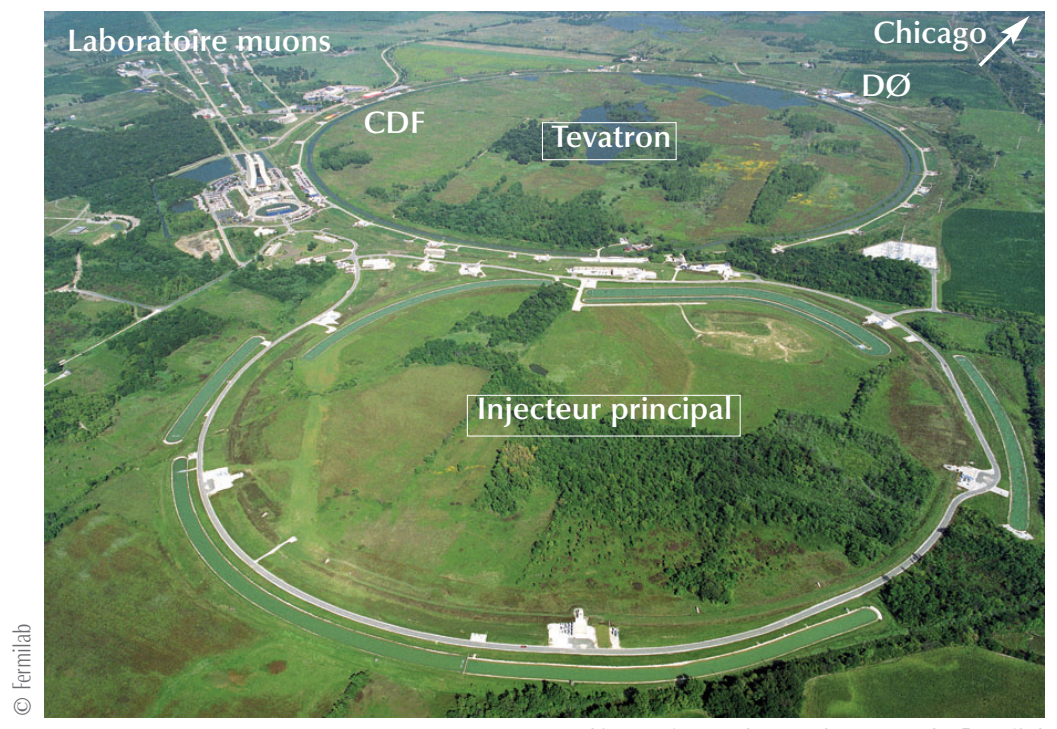

Vue aérienne des accélérateurs du Fermilab

Le Tevatron, principal équipement expérimental du Fermilab, a commencé à fonctionner en 1983, et est actuellement, en attendant le démarrage du LHC (voir article de B. Mansoulié, pp. 5-9), l'accélérateur de particules de plus haute énergie au monde.

C'est un collisionneur protonantiproton. Les antiprotons sont obtenus par collision de faisceaux de protons avec une cible métallique. Protons et antiprotons sont accélérés indépendamment à une vitesse proche de celle de la lumière, et se collisionnent (avec une énergie de 1,96 TeV dans le centre de masse) dans les deux expériences CDF et DØ.

Celles-ci font I'objet de collaborations internationales, composées d'environ 500 physiciens chacune (dont une cinquantaine de Français du CNRS et du CEA, qui travaillent en grande majorité dans $\mathrm{D} \varnothing)$.

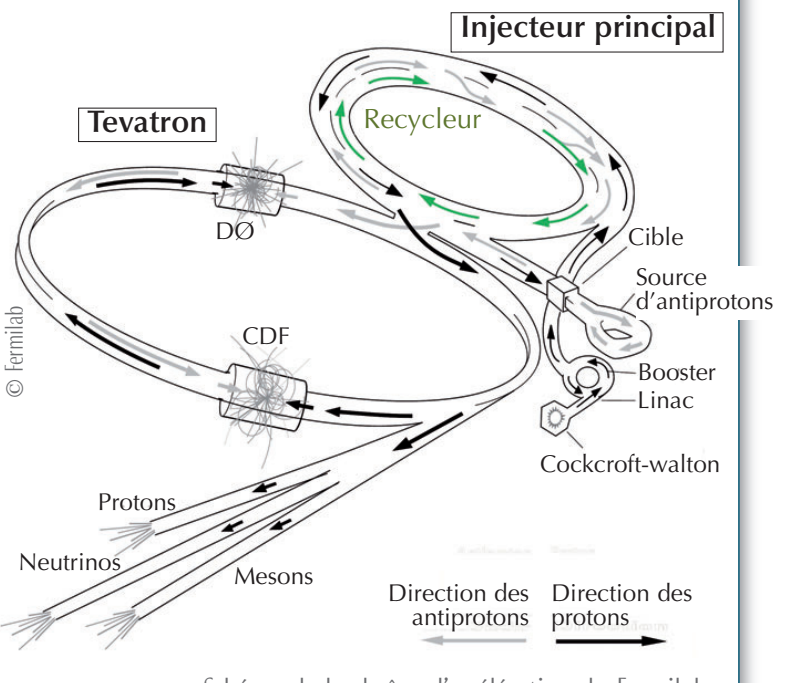

Schéma de la chaîne d’accélération du Fermilab

Le Tevatron a bénéficié en 1996-2001 d'un important programme d'amélioration, réalisé avec succès, et ses performances ont progressé de manière continue depuis 2003. 


\section{Production et désintégration du boson de Higgs}

Le Modèle Standard prévoit qu'un boson de Higgs $\mathrm{H}$ se désintègre préférentiellement en une paire quark-antiquark $\bar{b} \bar{b}$ s'il est "léger » $\left(\mathrm{m}_{\mathrm{H}}<135 \mathrm{GeV}\right)$, ou, s'il est plus lourd $\left(\mathrm{m}_{\mathrm{H}}>135 \mathrm{GeV}\right)$, en une paire de bosons W produisant un état final avec deux leptons et deux neutrinos. La stratégie de recherche au Tevatron dépend donc de la masse du boson de Higgs, et on distingue deux grands régimes : basse masse et haute masse.

La production du boson de Higgs peut se faire de manière directe, $p-\bar{p} \rightarrow \mathrm{H}$, comme l'indique la figure $1 \mathrm{a}$, ou en production associée avec un boson $\mathrm{W}$ ou $\mathrm{Z}$, $\mathrm{p}-\overline{\mathrm{p}} \rightarrow \mathrm{WH}$ (fig. $1 \mathrm{~b}$ ) ou $\mathrm{p}-\overline{\mathrm{p}} \rightarrow \mathrm{ZH}$ (fig. 1c, d). La production directe est exploitable à haute masse, car l'état final contient deux leptons et deux neutrinos, ce qui est suffisamment caractéristique pour pouvoir être bien identifié par rapport aux événements de bruit de fond. En revanche, à basse masse, seule la production associée WH ou $\mathrm{ZH}$ peut être séparée du bruit de fond, à l'aide des leptons de désintégration du W ou du Z et de la paire de quarks $b$ provenant du boson de Higgs. En fait, expérimentalement, on n'observe pas directement les quarks ou les gluons, mais les jets de hadrons (tels le pion ou le proton) qui se forment immédiatement par interaction forte dans la direction du quark ou du gluon produit.

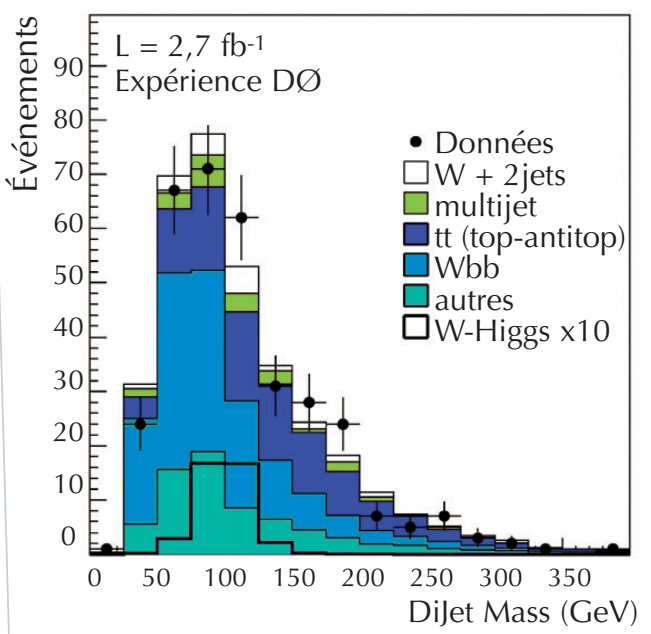

2. Distribution de la masse reconstruite à partir des deux jets de quark $\boldsymbol{b}$ (" DiJet Mass »), dans les événements $W+2$ jets de $b$ (fig. 1b). Les barres d'erreur sur les données sont d'origine statistique. La simulation des bruits de fond principaux provenant du Modèle Standard, telle la production qq $\rightarrow$ top-antitop ou Wb $\bar{b}$, additionnés aux autres bruits de fond simulés, reproduit bien les données. Le signal prédit dans le Modèle Standard pour un boson de Higgs de $115 \mathrm{GeV}$, et amplifié d'un facteur 10, est également représenté.
Au LHC, l'énergie dans le centre de masse est plus élevée. La recherche pourra être étendue jusqu'à des masses d'environ $1 \mathrm{TeV}$, dans le canal de désintégration $\mathrm{H} \rightarrow \mathrm{ZZ}$, qui offre peu de sensibilité au Tevatron, mais qui est le canal le plus clairement visible au LHC, si la masse du boson de Higgs est supérieure à environ $130 \mathrm{GeV}$. À basse masse aussi, la stratégie de recherche au LHC est différente de celle du Tevatron, puisque les bruits de fond empêchent une recherche efficace des modes de production WH et $\mathrm{ZH}$, avec $\mathrm{H} \rightarrow \bar{b} \bar{b}$. Dans ce cas, la recherche s'effectue prioritairement en détectant les désintégrations rares du Higgs en paires de photons, qui ont l'avantage de présenter un bruit de fond acceptable et de permettre une détermination précise de $\mathrm{m}_{\mathrm{H}}$.

\section{Recherche du boson de Higgs à basse masse $\left(m_{H}<135 \mathrm{Gel}\right)$}

À basse masse, plusieurs canaux de production-désintégration contribuent de manière importante. Le canal WH tout d'abord, mais aussi le canal ZH, dans lequel le $\mathrm{Z}$ se désintègre en paires de neutrinos, ou en paires de leptons chargés.

\section{Canal WH}

Dans ce canal (fig. 1b), on recherche les produits de désintégration des bosons W et Higgs, soit un lepton chargé, une paire de quarks $b \bar{b}$, et de l'impulsion " transverse " manquante due au neutrino (qui n'est pas détecté). En effet, comme les détecteurs ne sont pas totalement hermétiques dans les régions proches de l'axe du faisceau, définissant l'axe longitudinal, on ne peut mesurer précisément l'impulsion manquante que dans le plan transversal. Celle-ci est mesurée soit à partir de l'énergie déposée par les particules dans le calorimètre, soit en déterminant l'impulsion des traces chargées par la mesure de la courbure de leur direction dans le champ magnétique (voir encadré, p. 8). L'enregistrement des événements candidats s'effectue lorsque le système de déclenchement détecte un lepton de haute énergie ou lorsqu'un lepton est détecté en même temps qu'un ou plusieurs jets de particules. Une première sélection hors-ligne des événements enregistrés est ensuite effectuée, réduisant leur nombre de 30 millions à environ 65 000. À ce stade, on vérifie que la simulation du bruit de fond décrit bien ces événements, alors que la quantité d'événements W-Higgs attendue (une trentaine) est négligeable par rapport aux événements de bruit de fond.
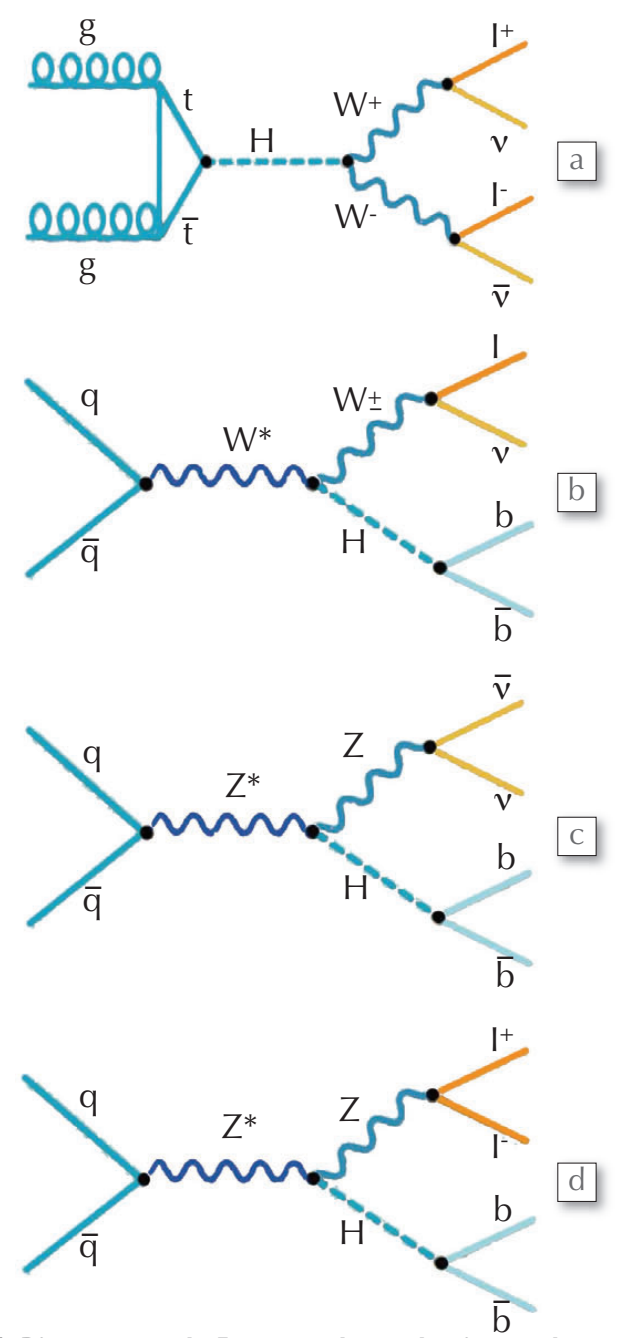

1. Diagrammes de Feynman de production et de désintégration du boson de Higgs au Tevatron : a) gluon-gluon $\rightarrow \mathrm{H} \rightarrow \mathrm{WW}$; b, c, d) quark-antiquark $\rightarrow$ WH ou $Z \mathrm{H}$, avec $\mathrm{H} \rightarrow b \bar{b}$. Les gluons, quarks et antiquarks à la gauche des diagrammes proviennent du proton et de l'antiproton qui entrent en collision. Les bosons W et $Z$ se désintègrent en leptons chargés $\left(t^{+} /\right)$et/ou en neutrinos $(v)$, comme indiqué. $W^{*}$ et $Z^{*}$ sont des bosons $W$ et $Z$ virtuels.

Pour devenir sensible aux événements " Higgs ", on identifie les jets de quarks $b$ provenant de leur désintégration, en utilisant des algorithmes dédiés. Dans la sélection finale (lepton +2 jets de $b+$ impulsion transverse manquante), on retient alors environ 350 événements, et environ quatre d'entre eux contiennent un boson de Higgs (s'il pèse $115 \mathrm{GeV}$ ), qui se désintègre en paires de quarks $b$. En faisant la distribution de la masse reconstruite à partir des particules provenant des quarks $b$, on peut donc rechercher un excès d'événements à une masse donnée. Cette distribution est représentée dans la figure 2, avec les différents bruits de fond du Modèle Standard, définis dans la légende, qui s'additionnent pour décrire correctement les données. Le signal attendu d'un boson de Higgs ayant une masse de $115 \mathrm{GeV}$ est également représenté sur la figure, amplifié d'un facteur 10, ce qui montre la difficulté à extraire un signal aussi petit. 
Nous utilisons donc des techniques avancées (tels des algorithmes dits «à réseaux de neurones ") pour augmenter le rapport du signal WH sur le bruit de fond du Modèle Standard. Mais, pour mettre en évidence un boson de Higgs léger, il faudra encore améliorer la discrimination signal/ bruit, augmenter le nombre d'événements analysés et combiner ce type de production avec d'autres, décrits ci-dessous.

\section{Canal 개}

Ce canal (fig. 1c et d) a deux manifestations très différentes, suivant que le boson $\mathrm{Z}$ se désintègre en une paire de leptons chargés ou en une paire de neutrinos.

Le canal $\mathrm{ZH} \rightarrow 1^{+} 1^{-} \bar{b}$ (fig. 1d) a un faible taux de production, car le taux de désintégration du $\mathrm{Z}$ en leptons chargés n'est que de 3\% par type de lepton. Mais ceci est compensé par l'excellente définition de l'état final : deux leptons facilement identifiables, deux jets de quarks $b$. Tout l'état final étant reconstruit, il y a peu d'incertitude provenant d'une impulsion transverse manquante. Avec ces caractéristiques, la réjection du bruit de fond est très efficace, et permet alors de compenser en partie la faiblesse de la production initiale.

Le canal $\mathrm{ZH} \rightarrow \boldsymbol{v} \overline{\mathbf{v}} \bar{b}$ (fig. 1c) est analysé de manière très différente, puisque les neutrinos ne laissent pas de traces dans les détecteurs ; leur production ne peut donc être déduite que par la présence d'une grande impulsion transverse manquante. Le déclenchement de l'enregistrement des événements candidats se base sur l'impulsion transverse manquante des jets, ce qui ne permet pas d'éviter de garder un grand nombre d'événements de bruit de fond produits par interaction forte. Une fois analysé, ce canal a une sensibilité semblable à celle du canal WH.

\section{Recherche du boson de Higgs à haute masse $\left(m_{H}>135 \mathrm{Gel}\right)$}

La recherche à haute masse est effectuée en utilisant principalement la désintégration $\mathrm{H} \rightarrow \mathrm{W}^{+} \mathrm{W}^{-}$(fig. 1a). Cette analyse tire profit du caractère scalaire du boson de Higgs : le système $\mathrm{W}^{+} \mathrm{W}$ - étant produit dans un état de spin 0, les leptons chargés issus des désintégrations des deux bosons W sont préférentiellement alignés dans une direction donnée, alors que les neutrinos le sont dans la direction opposée, ce qui produit une impulsion manquante importante dans le bilan énergie-impulsion.

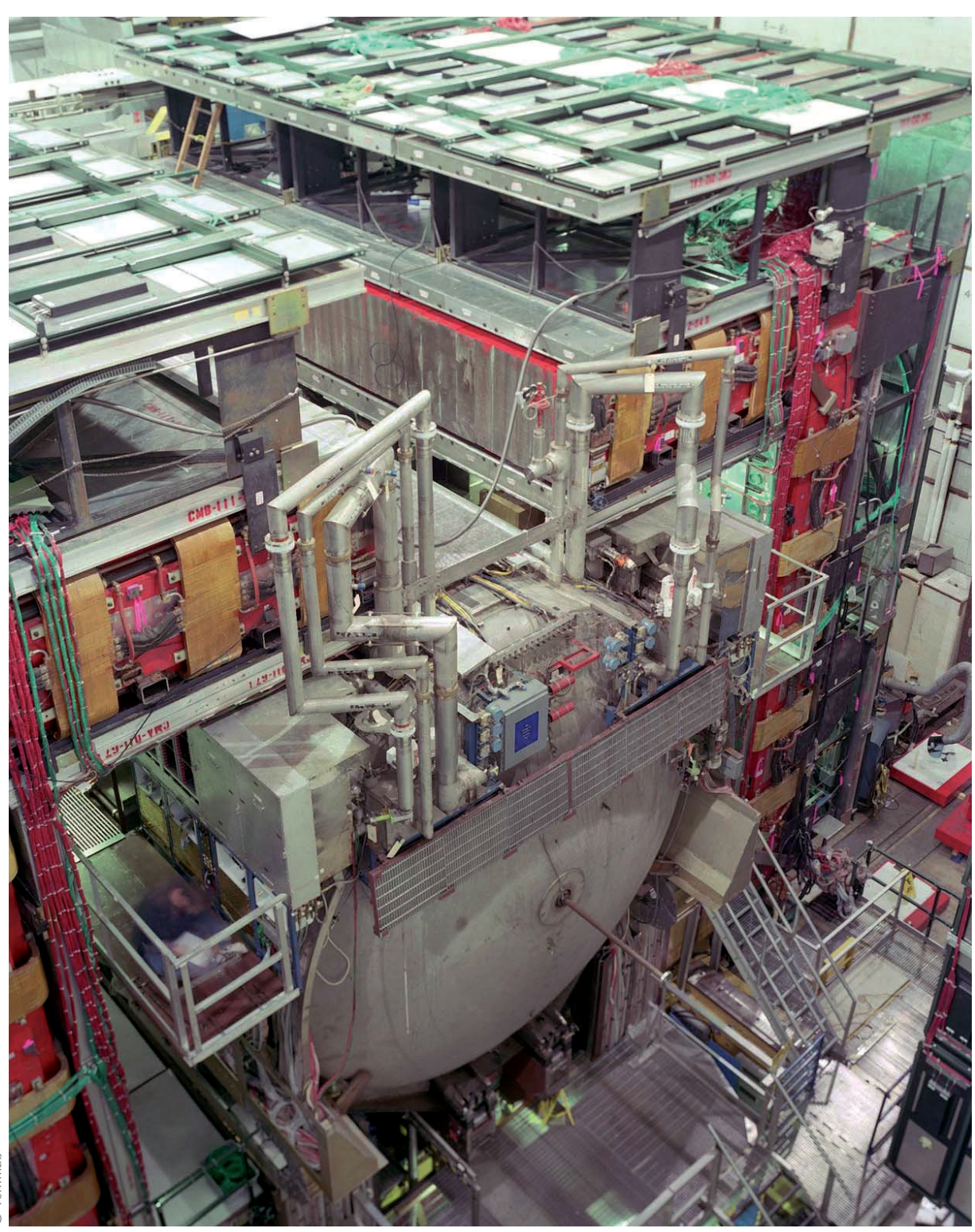

Le détecteur DØ. On voit les chambres à muons (plates, extérieures, en haut de la photo) entourant le cryostat cylindrique qui contient le calorimètre à argon liquide.

L'analyse va donc rechercher un état final avec deux leptons de charges opposées et une grande impulsion transverse manquante. Les événements candidats sont enregistrés lorsqu'un dépôt d'énergie caractéristique d'un électron ou lorsqu'une trace chargée typique d'un muon, sont détectés dans le système de déclenchement. Ils sont ensuite sélectionnés afin de reproduire les caractéristiques d'une désintégration de boson de Higgs, tout en rajoutant des conditions supplémentaires pour rejeter autant que possible le bruit de fond. Après cette sélection, l'expérience DØ a isolé environ 130 événements candidats, et si le boson de Higgs a une masse de $165 \mathrm{GeV}$, une quinzaine de ces événements seraient les produits de sa désintégration.

Il nous reste alors à séparer ces événements de signal de ceux du bruit de fond ; pour cela les deux expériences D $\varnothing$ et CDF utilisent des techniques d'analyses avancées, qui se servent des variables cinématiques des événements de bruit de fond $\bar{p} \bar{p} \rightarrow \mathrm{W}^{+} \mathrm{W}^{-}$, et de signal $\mathrm{p} \overline{\mathrm{p}} \rightarrow \mathrm{H} \rightarrow \mathrm{W}^{+} \mathrm{W}^{-}$.

\section{Combinaison des différents canaux de recherche du boson de Higgs}

Chaque canal de production étant statistiquement limité, les deux expériences combinent les résultats de tous leurs canaux pour obtenir la meilleure sensibilité possible. Cette combinaison utilise des techniques statistiques bien établies, et est faite en tenant compte des incertitudes statistiques et systématiques. Le résultat de cette combinaison, obtenu en mars 2009 avec en moyenne 1/3 des données (de 0,9 à $4,2 \mathrm{fb}^{-1}$ suivant les canaux) attendues à la fin de 2010, est représenté dans la figure 3 en fonction de la masse du boson de Higgs, sous la forme de la limite supérieure actuelle de la section efficace de production du boson de Higgs divisée par la valeur de cette même section efficace prédite dans le Modèle Standard. Un rapport Tevatron attendu inférieur à 1 signifie que l'expérience a atteint une sensibilité suffisante pour commencer à détecter le boson de Higgs. Un rapport Tevatron mesuré inférieur 
Tevatron Run II préliminaire, $L=0,9-4,2 \mathrm{fb}^{-1}$

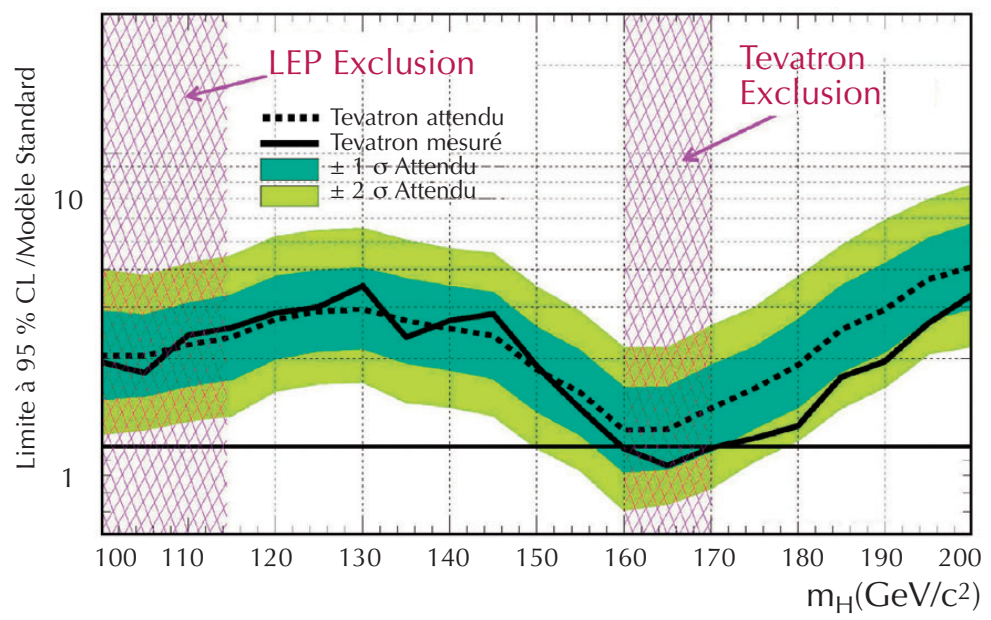

3. Limite supérieure (à 95\% C.L.) de la section efficace de production du boson de Higgs, normalisée par la valeur prédite pour cette section efficace dans le Modèle Standard, en fonction de la masse du boson de Higgs recherché. Les valeurs mesurées (trait plein) et attendues en supposant l'absence de signal Higgs et de fluctuations statistiques dans les données (trait pointillé) sont représentées, ainsi que les dispersions statistiques possibles sur la limite attendue (bandes de couleurs à 1 ou 2 écarts standards).
Tevatron Run II préliminaire, $\mathrm{L}=\mathbf{0}, \mathbf{9 - 4 , 2} \mathrm{fb}-\mathbf{1}$

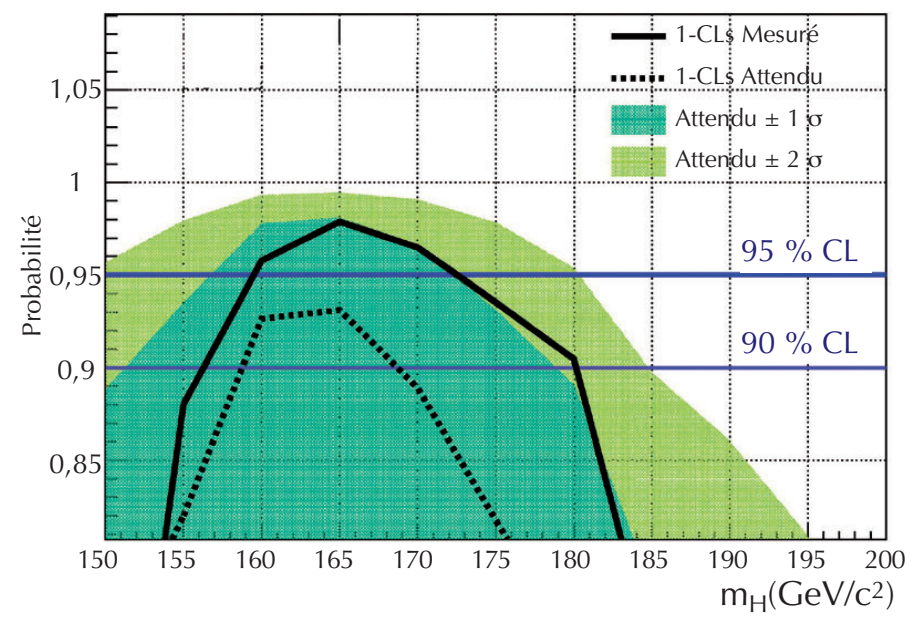

4. Résultats de la combinaison CDF-DØ : probabilité que le boson de Higgs n'ait pas la valeur de masse en abscisse. On considère que si la probabilité dépasse $95 \%$, la valeur est exclue, ce qui est le cas ici pour $m_{H}$ entre 160 et $170 \mathrm{GeV}$. On a représenté ici les valeurs observées (trait plein) et les valeurs attendues en supposant l'absence de signal Higgs et de fluctuations statistiques dans les données (trait pointillé), ainsi que les dispersions statistiques sur les valeurs attendues (bandes de couleurs à 1 ou 2 écarts standards). à 1 pour une masse donnée indiquerait que le boson de Higgs n'a pas la masse en question. Un rapport Tevatron mesuré significativement supérieur à Tevatron attendu pour une masse donnée serait en faveur d'un boson de Higgs de cette masse, si la valeur Tevatron attendu est inférieure à 1 . Dans la figure 3, on observe qu'à basse masse $(115 \mathrm{GeV})$, la sensibilité actuelle se situe environ deux fois au-dessus de la prédiction du Modèle Standard et nécessite donc plus de données et des progrès supplémentaires dans les techniques d'analyses pour pouvoir être sensible à un boson de Higgs léger. En revanche, à $165 \mathrm{GeV}$, le rapport observé est de 0,8 , donc l'hypothèse que le boson de Higgs ait une masse de $165 \mathrm{GeV}$ est exclue. On constate aussi qu'avec cette quantité de données, si le boson de Higgs n'avait pas cette masse, on se serait attendu à un rapport un peu plus élevé, de l'ordre de 1,1 ; mais ceci reste tout à fait compatible avec l'incertitude statistique du résultat, comme le montrent les bandes colorées. On voit aussi sur la figure 3 que l'exclusion s'étend à l'intervalle 160-170 GeV.

Pour estimer quelle est la probabilité d'exclusion à haute masse, on peut représenter ce résultat d'une autre façon, comme le montre la figure 4. On constate alors que la région à plus haute masse $(170-180 \mathrm{GeV})$ a aussi une forte probabilité d'exclusion, ce qui implique, en utilisant les contraintes indirectes discutées dans l'introduction, que le boson de Higgs a très probablement une masse bien inférieure à $160 \mathrm{GeV}$. C'est la première fois depuis l'arrêt du LEP en
2001 que de nouvelles contraintes directes sont apportées sur la masse du boson de Higgs. Ce résultat démontre que le Tevatron peut maintenant rechercher activement cette particule, en augmentant la quantité de données analysées.

\section{Perspectives de découverte du boson de Higgs}

Les groupes de recherche du boson de Higgs au Tevatron sont en train de redoubler d'efforts pour analyser les nouvelles données enregistrées et pour améliorer encore leurs techniques d'analyse. Ils comptent en particulier sur une amélioration de la séparation signal/bruit par des développements supplémentaires des techniques avancées et, à basse masse, sur une amélioration de l'efficacité d'identification des jets de quarks $b$ et un gain en résolution de la masse invariante du système $\bar{b} \bar{b}$. Le gain global attendu en sensibilité a été quantifié et, avec ces améliorations et la quantité finale de données attendue, tout le domaine entre 115 et $185 \mathrm{GeV}$ pourra être sondé. Un scénario particulièrement étonnant serait, par exemple, une exclusion du boson de Higgs dans tout ce domaine, ce qui serait en contradiction avec les contraintes décrites dans l'introduction de cet article, et donc remettrait en cause le mécanisme de Higgs du Modèle Standard.

Une observation à trois écarts standards devrait être possible. Cela dépendra in fine des performances atteintes par les analyses et par la machine, et par la durée de la prise de données qui pourrait être prolongée jusqu'en 2011. Même si la découverte définitive du boson de Higgs n'était faite qu'au LHC, les recherches au Tevatron sont cependant cruciales pour la compréhension de la nature du boson de Higgs, en particulier s'il est de basse masse. Dans ce cas, il ne pourra pas être observé avant longtemps au LHC dans son mode de désintégration principal $\mathrm{H} \rightarrow \bar{b}$, à cause du bruit de fond prohibitif, mais pourra l'être par ses désintégrations rares, en particulier en photonphoton. L'observation au Tevatron de la désintégration en $\bar{b} \bar{b}$ renforcerait alors l'hypothèse que la particule observée est bien le boson de Higgs du Modèle Standard. Les efforts des équipes françaises engagées sur ce projet depuis une décennie seraient ainsi récompensés, et ce résultat apporterait une avancée fondamentale dans la compréhension de la brisure de la symétrie électrofaible.

\section{Pour en savoir plus...}

- V. RuhImann-Kleider, Reflets de la physique 12 (décembre 2008-janvier 2009) 16-19.

2 - Pour un panorama plus complet, tant théorique qu'expérimental, de la physique du boson de Higgs du Modèle Standard et au-delà, consulter : G. Bernardi, M. Carena et T. Junk, Higgs bosons: theory and searches, Particle Data Review 2007-2008, http://pdg./bl.gov/2007/reviews/higgs_s055.pdf

- Pour une approche plus abordable : M. Grousson, "Boson de Higgs, "La particule de Dieu" à portée de main ", Science et Vie, n⿳1088 (mai 2008) 54-70.

- M. Davier, LHC : Enquête sur le boson de Higgs, Éditions du Pommier (2008). 